\title{
Synthesis and Biological Activities of 2-Substituted Benzimidazole-Metal Complexes
}

\author{
Md. Afzal Azam, B. R. P. Kumar, R. Mazumdar and B. Suresh \\ Department of Pharmaceutical Chemistry, J. S. S., College of Pharmacy, \\ Ootacamund-643001, Tamil Nadu, India
}

\begin{abstract}
A series of copper(II) and cobalt(II) coordination compounds with 2-substituted benzimidazole derived monodentate and bidentate ligands have been prepared and characterized by microanalysis, IR and UV-Vis spectroscopy. Synthesized metal complexes have been screened for their in vitro antioxidant and antitumor activity. The complex $4 \mathbf{a}$ showed significant nitric oxide free radical scavenging activity $\left(\mathrm{IC}_{50} 65 \mu \mathrm{g} / \mathrm{ml}\right)$, while $3 \mathbf{i}$ and $3 \mathrm{~g}$ showed potent superoxide dismutase activity with $\mathrm{IC}_{50}$ of 0.26 and $0.28 \mu \mathrm{M}$ respectively. In vitro cytotoxicity study with human breast MCF-7 and CNS SF 268 cancer cell lines showed that the most active 2benzyl-1H-benzimidazole $\mathrm{Cu}$ (II) complex 3a inhibited the growth of cancer cells at $20 \mu \mathrm{M}$ concentration.
\end{abstract}

Keywords: $\mathrm{Cu}(\mathrm{II})$ complexes, $\mathrm{Co}(\mathrm{II})$ complexes, Benzimidazoles, Antitumor activity.

\section{INTRODUCTION}

2-Substituted benzimidazole derivatives are an important class of compounds in medicinal and organic chemistry. The benzimidazole moiety is a structural element of compounds with a wide range of biological activities ${ }^{1-4}$. For example, 2-substituted benzimidazoles exhibit inhibitory activity against a range of human tumour cell lines in vitro. ${ }^{5-7}$ In addition transition metal complexes of 2-substituted benzimidazole ligands act as anticancer agents ${ }^{8-12}$. Cancer cells are found to have less superoxide dismutase activity than normal cells $\mathrm{s}^{13,14}$ and copper(II) complexes are known to mimic activity of copper, zinc-superoxide dismutase (Cu,Zn-SOD), an antioxidant enzyme that protect cells from the toxic effect of superoxide ion by its dismutation into dioxygen and hydrogen peroxide in biological systems ${ }^{15}$. The antitumor activity of SOD metal complexes has been suggested to be due to their superoxide scavenging ability ${ }^{16}$. Above observations

Correspondence to: Md. Afzal Azam

E-mail: afzal9azam@hotmail.com

Fax: +91-423-2442937.

Dhaka Univ. J. Pharm. Sci. 8(2): 131-140, 2009 (December) prompted us to synthesize various 2-substituted benzimidazole-copper(II) and cobalt(II) complexes 3a-i and 4a-i as per Scheme 1 with a view to evaluate their anticancer and antioxidant activities.

\section{MATERIALS AND METHODS}

Melting points were obtained in an open capillary tube. Structures of all the compounds were confirmed by elemental analysis and spectral data. The IR spectra were recorded on Shimadzu 800 FTIR spectrometer in $\mathrm{KBr}$ pellets. The ${ }^{1} \mathrm{H}$ NMR spectra were recorded on a Perkin-Elmer R-32 (90 MHz) spectrometer in $\mathrm{CDCl}_{3}$ using TMS as an internal standard. All chemical shift values are expressed in $\delta$ ppm. Mass spectra were recorded on a Shimadzu 2010A LC-MS spectrometer and UV spectra were taken on Perkin Elmer version 2.5 spectrophotometer. Elemental analysis of compounds was carried out on Flash EA 1112 series instrument. The homogeneity of all the compounds were verified on silica gel G coated TLC plates and the spots were located by iodine vapours. 


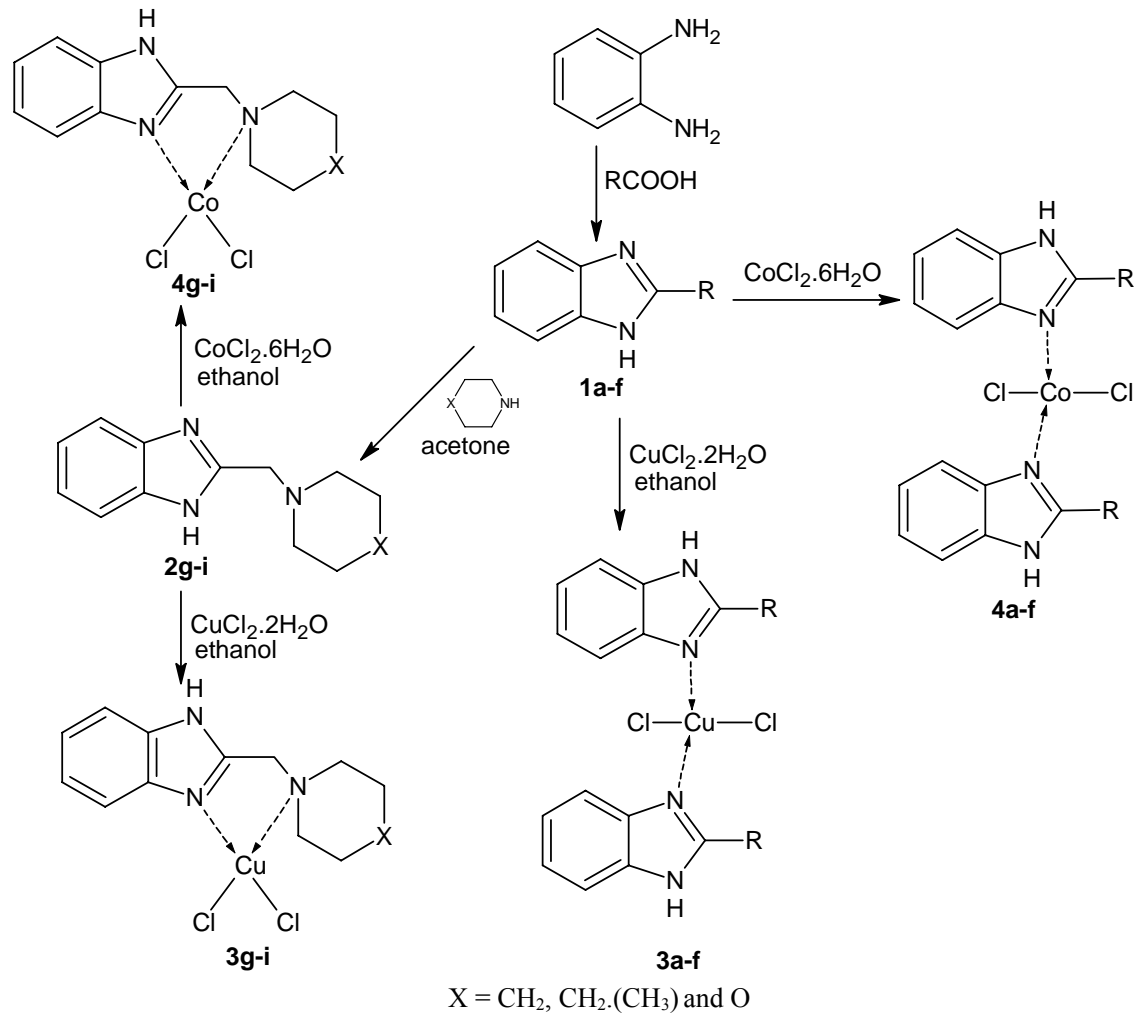

$\mathrm{R}=\mathrm{CH}_{2} \cdot \mathrm{C}_{6} \mathrm{H}_{5}, \mathrm{SH}, \mathrm{S} \cdot \mathrm{CH}_{2} \cdot \mathrm{C}_{6} \mathrm{H}_{5}, \mathrm{C}_{6} \mathrm{H}_{5} \cdot \mathrm{NH}_{2}, \mathrm{CH}_{2} \mathrm{Cl}$ and $\mathrm{C}_{6} \mathrm{H}_{5}$

Scheme 1: Synthesis of 2-substituted benzimidazole-metal complexes

Table 1. Characterization data of synthesized compounds 1a-f, 2g-i, 4a-i and 5a-i

\begin{tabular}{|c|c|c|c|c|c|}
\hline Compound & (R) & $\mathrm{X}$ & Formula & $\mathrm{MP}\left({ }^{0} \mathrm{C}\right)$ & *Yield (\%) \\
\hline $1 a$ & $\mathrm{CH}_{2} \mathrm{C}_{6} \mathrm{H}_{5}$ & --- & $\mathrm{C}_{14} \mathrm{H}_{12} \mathrm{~N}_{2}$ & $191^{17}$ & 59 \\
\hline $1 b$ & $\mathrm{CH}_{2} \mathrm{Cl}$ & --- & $\mathrm{C}_{8} \mathrm{H}_{7} \mathrm{ClN}_{2}$ & $147^{22}$ & 65 \\
\hline 1c & $\mathrm{C}_{6} \mathrm{H}_{4} \mathrm{NH}_{2}$ & --- & $\mathrm{C}_{13} \mathrm{H}_{11} \mathrm{~N}_{3}$ & 222 & 68 \\
\hline 1d & $\mathrm{C}_{6} \mathrm{H}_{5}$ & --- & $\mathrm{C}_{13} \mathrm{H}_{10} \mathrm{~N}_{2}$ & $295^{22}$ & 71 \\
\hline $1 \mathrm{e}$ & $\mathrm{SH}$ & --- & $\mathrm{C}_{7} \mathrm{H}_{6} \mathrm{~N}_{2} \mathrm{~S}$ & $302^{22}$ & 73 \\
\hline 1f & S. $\mathrm{CH}_{2} \mathrm{C}_{6} \mathrm{H}_{5}$ & -- & $\mathrm{C}_{14} \mathrm{H}_{12} \mathrm{~N}_{2} \mathrm{~S}$ & 194 & 69 \\
\hline $2 \mathrm{~g}$ & --- & $\mathrm{CH}_{2}$ & $\mathrm{C}_{13} \mathrm{H}_{17} \mathrm{~N}_{3}$ & 235 & 62 \\
\hline $2 h$ & -- & $\mathrm{CH}-\mathrm{CH}_{3}$ & $\mathrm{C}_{14} \mathrm{H}_{19} \mathrm{~N}_{3}$ & 263 & 70 \\
\hline $2 \mathbf{i}$ & --- & $-\mathrm{O}-$ & $\mathrm{C}_{12} \mathrm{H}_{15} \mathrm{~N}_{3} \mathrm{O}$ & 233 & 64 \\
\hline $4 a$ & $\mathrm{CH}_{2} \mathrm{C}_{6} \mathrm{H}_{5}$ & --- & $\mathrm{C}_{28} \mathrm{H}_{24} \mathrm{Cl}_{2} \mathrm{CuN}_{4}$ & 150 & 63 \\
\hline $4 b$ & $\mathrm{CH}_{2} \mathrm{Cl}$ & --- & $\mathrm{C}_{16} \mathrm{H}_{14} \mathrm{Cl}_{4} \mathrm{CuN}_{4}$ & 170 & 65 \\
\hline $4 c$ & $\mathrm{C}_{6} \mathrm{H}_{4} \cdot \mathrm{NH}_{2}$ & --- & $\mathrm{C}_{26} \mathrm{H}_{22} \mathrm{Cl}_{2} \mathrm{CuN}_{6}$ & 48 & 77 \\
\hline $4 d$ & $\mathrm{C}_{6} \mathrm{H}_{5}$ & --- & $\mathrm{C}_{26} \mathrm{H}_{20} \mathrm{Cl}_{2} \mathrm{CuN}_{4}$ & 230 & 71 \\
\hline $4 e$ & SH & --- & $\mathrm{C}_{14} \mathrm{H}_{12} \mathrm{Cl}_{2} \mathrm{CuN}_{4} \mathrm{~S}_{2}$ & 72 & 65 \\
\hline 4f & S. $\mathrm{CH}_{2} \mathrm{C}_{6} \mathrm{H}_{5}$ & $\ldots$ & $\mathrm{C}_{28} \mathrm{H}_{24} \mathrm{Cl}_{2} \mathrm{CuN}_{4} \mathrm{~S}_{2}$ & 120 & 67 \\
\hline $4 \mathrm{~g}$ & --- & $\mathrm{CH}_{2}$ & $\mathrm{C}_{13} \mathrm{H}_{17} \mathrm{Cl}_{2} \mathrm{CuN}_{3}$ & 99 & 62 \\
\hline $4 h$ & --- & $\mathrm{CH}-\mathrm{CH}_{3}$ & $\mathrm{C}_{14} \mathrm{H}_{19} \mathrm{Cl}_{2} \mathrm{CuN}_{3}$ & 136 & 66 \\
\hline $4 i$ & --- & $-\mathrm{O}-$ & $\mathrm{C}_{12} \mathrm{H}_{15} \mathrm{Cl}_{2} \mathrm{CuN}_{3} \mathrm{O}$ & 123 & 64 \\
\hline 3a & $\mathrm{CH}_{2} \mathrm{C}_{6} \mathrm{H}_{5}$ & --. & $\mathrm{C}_{28} \mathrm{H}_{24} \mathrm{Cl}_{2} \mathrm{CoN}_{4}$ & 237 & 79 \\
\hline $3 \mathbf{b}$ & $\mathrm{CH}_{2} \mathrm{Cl}$ & --- & $\mathrm{C}_{16} \mathrm{H}_{14} \mathrm{Cl}_{4} \mathrm{CoN}_{4}$ & 190 & 71 \\
\hline $3 c$ & $\mathrm{C}_{6} \mathrm{H}_{4} \mathrm{NH}_{2}$ & ... & $\mathrm{C}_{26} \mathrm{H}_{22} \mathrm{Cl}_{2} \mathrm{CoN}_{6}$ & 70 & 71 \\
\hline 3d & $\mathrm{C}_{6} \mathrm{H}_{5}$ & --- & $\mathrm{C}_{26} \mathrm{H}_{20} \mathrm{Cl}_{2} \mathrm{CoN}_{4}$ & 150 & 67 \\
\hline $3 e$ & $\mathrm{SH}$ & --- & $\mathrm{C}_{14} \mathrm{H}_{12} \mathrm{Cl}_{2} \mathrm{CoN}_{4} \mathrm{~S}_{2}$ & 45 & 65 \\
\hline $3 f$ & S. $\mathrm{CH}_{2} \mathrm{C}_{6} \mathrm{H}_{5}$ & $\ldots$ & $\mathrm{C}_{28} \mathrm{H}_{24} \mathrm{Cl}_{2} \mathrm{CoN}_{4} \mathrm{~S}_{2}$ & 200 & 63 \\
\hline $3 g$ & --- & $\mathrm{CH}_{2}$ & $\mathrm{C}_{13} \mathrm{H}_{17} \mathrm{Cl}_{2} \mathrm{CoN}_{3}$ & 136 & 62 \\
\hline $3 \mathbf{h}$ & --- & $\mathrm{CH}-\mathrm{CH}_{3}$ & $\mathrm{C}_{14} \mathrm{H}_{19} \mathrm{Cl}_{2} \mathrm{CoN}_{3}$ & 198 & 61 \\
\hline $3 \mathbf{i}$ & --- & -O- & $\mathrm{C}_{12} \mathrm{H}_{15} \mathrm{Cl}_{2} \mathrm{CoN}_{3} \mathrm{O}$ & 172 & 64 \\
\hline
\end{tabular}

* Isolated yield. All compounds showed satisfactory elemental analysis. ${ }^{17,22}$ Lit. M.P. in ${ }^{0}$ C 1a: 191; 1b: 146-148; 1d: 293-296; 1e: 300-304. 
Chemistry. Synthesis of 2-substituted-1Hbenzimidazoles 1a-f was carried out according to the standard procedure ${ }^{17}$ and characterization data is presented in Table 1.

1c: IR $\left(\mathrm{KBr}, \mathrm{cm}^{-1}\right)$ : 3420, $3360(\mathrm{~N}-\mathrm{H}), 3046$ $(\mathrm{Ar}-\mathrm{H}), 1635(\mathrm{C}=\mathrm{N}), 1606(\mathrm{C}=\mathrm{C}$ aromatic $), 1265(\mathrm{C}-$ N). ${ }^{1} \mathrm{H}$ NMR $(\delta \mathrm{ppm})\left(\mathrm{CDCl}_{3}\right): \delta 7.8(\mathrm{~s}, 1 \mathrm{H}, \mathrm{NH}), 7.6$ (s, 2H, NH), 6.9-7.1 (m, 8H, Ar-H). MS: m/z 209 $\left(\mathrm{M}^{+}\right)$. Calculated for $\mathrm{C}_{13} \mathrm{H}_{11} \mathrm{~N}_{3}: 74.64 \% \mathrm{C} ; 5.26 \%$ H; $20.09 \%$ N..Found: $74.68 \%$ C; $5.23 \%$ H; $20.05 \%$ N; 1f: IR (KBr, $\left.\mathrm{cm}^{-1}\right)$ : $3448(\mathrm{~N}-\mathrm{H}), 3072(\mathrm{Ar}-\mathrm{H})$, 2958 (C-H aliphatic), $1652(\mathrm{C}=\mathrm{N}), 1584(\mathrm{C}=\mathrm{C}$ aromatic), $1268 \quad(\mathrm{C}-\mathrm{N}), \quad 749$ (monosubstituted benzene). ${ }^{1} \mathrm{H}$ NMR $(\delta \mathrm{ppm})\left(\mathrm{CDCl}_{3}\right): \delta 7.6(\mathrm{~s}, 1 \mathrm{H}$, NH) 6.8-7.3 (m, 9H, Ar-H), $4.2\left(\mathrm{~s}, 2 \mathrm{H}, \mathrm{CH}_{2}\right)$. MS: $\mathrm{m} / \mathrm{z} 240\left(\mathrm{M}^{+}\right)$. Calculated for $\mathrm{C}_{14} \mathrm{H}_{12} \mathrm{~N}_{2} \mathrm{~S}: 70.00 \% \mathrm{C}$; $5.00 \%$ H; $11.66 \%$ N. Found: $70.03 \%$ C; $4.98 \%$ H; $11.68 \% \mathrm{~N}$.

Synthesis of 2-(alkyl/arylamine-1-ylmethyl)-1Hbenzimidazoles $\mathbf{2 g}$-i: To a mixture of 2-chloromethyl benzimidazole $1 \mathbf{b}(0.01 \mathrm{~mol})$ and appropriate amine $(0.01 \mathrm{~mol})$ in $50 \mathrm{ml}$ of dry acetone, anhydrous potassium carbonate $(0.69 \mathrm{~g}, 0.005 \mathrm{~mol})$ was added. The reaction mixture was stirred at room temperature for $10-12 \mathrm{~h}$. The solid thus obtained was filtered, washed with water, dried and recrystallized from acetone: methanol (1:1); $2 \mathrm{~g}$ : IR $\left(\mathrm{KBr}, \mathrm{cm}^{-1}\right): 3340$ (N-H), 3060 (Ar-H ), 2936, 2880 (C-H aliphatic), $1654(\mathrm{C}=\mathrm{N}), 1618(\mathrm{C}=\mathrm{C}$ aromatic $), 1266(\mathrm{C}-\mathrm{N}) ;{ }^{1} \mathrm{H}$ $\mathrm{NMR}(\delta \mathrm{ppm})\left(\mathrm{CDCl}_{3}\right): \delta 7.72(\mathrm{~s}, 1 \mathrm{H}, \mathrm{NH}), 6.85-7.63$ (m, 4H, ArH), $3.65\left(\mathrm{~s}, 2 \mathrm{H}, \mathrm{CH}_{2}\right), 2.80\left(\mathrm{~m}, 4 \mathrm{H}, 2 \mathrm{xCH}_{2}\right.$ piperidine), $1.92\left(\mathrm{~m}, 6 \mathrm{H}, 3 \mathrm{xCH}_{2}\right.$ piperidine). $\mathrm{MS}: \mathrm{m} / \mathrm{z}$ $215\left(\mathrm{M}^{+}\right)$. Calculated for $\mathrm{C}_{13} \mathrm{H}_{17} \mathrm{~N}_{3}: 72.55 \% \mathrm{C} ; 7.90$ $\% \mathrm{H} ; 19.53 \% \mathrm{~N}$. Found: $72.57 \% \mathrm{C} ; 7.93 \% \mathrm{H} ; 19.51$ $\%$ N; 2h: IR (KBr, cm $\left.{ }^{-1}\right): 3442(\mathrm{~N}-\mathrm{H}), 3025(\mathrm{Ar}-\mathrm{H})$, 2921, 2862 (C-H aliphatic), $1665(\mathrm{C}=\mathrm{N}), 1588(\mathrm{C}=\mathrm{C}$ aromatic), $1253 \quad(\mathrm{C}-\mathrm{N}) ; \quad \mathrm{MS}: \mathrm{m} / \mathrm{z} 229 \quad\left(\mathrm{M}^{+}\right)$. Calculated for $\mathrm{C}_{14} \mathrm{H}_{19} \mathrm{~N}_{3}: 73.36 \% \mathrm{C} ; 8.29 \% \mathrm{H}$; $18.34 \%$ N. Found: $73.38 \%$ C; $8.31 \% \mathrm{H} ; 18.35 \% \mathrm{~N}$; 2i: IR (KBr, $\left.\mathrm{cm}^{-1}\right)$ : 3454 (N-H), $3042(\mathrm{Ar}-\mathrm{H}), 2962$, 2862 (C-H aliphatic), $1647(\mathrm{C}=\mathrm{N}), 1595(\mathrm{C}=\mathrm{C}$ aromatic), 1268 (C-N), 1116 (C-O-C); MS: m/z 217 $\left(\mathrm{M}^{+}\right)$. Calculated for $\mathrm{C}_{12} \mathrm{H}_{15} \mathrm{~N}_{3} \mathrm{O}: 66.35 \% \mathrm{C} ; 6.91 \%$
H; $19.35 \%$ N. Found: $66.33 \%$ C; $6.92 \%$ H; $19.37 \%$ $\mathrm{N}$.

Synthesis of Dichlorobis[2-(alkyl/aryl/aralkyl/ thioaralkyl-1ylmethyl)-1H-benzimidazoles] copper (II) 3a-f: Appropriate benzimidazole ligand 1a-f $(0.01 \mathrm{~mol})$ was dissolved in ethanol $(50 \mathrm{ml})$ and the resulting solution was treated with copper(II) chloride $(0.005 \mathrm{~mol})$ dissolved in dimethylformamide $(2 \mathrm{ml})$. The reaction mixture was stirred at room temperature for $6 \mathrm{~h}$. The solid that separated in each case was filtered, washed with ether, dried and recrystallized from dimethylformamide; 3a: $\mathrm{IR}(\mathrm{KBr}$, $\left.\mathrm{cm}^{-1}\right): 3325$ (N-H), 3015 (Ar-H ), 2890 (C-H aliphatic), $1632(\mathrm{C}=\mathrm{N}), 1610(\mathrm{C}=\mathrm{C}$ aromatic $), 1272$ (C-N), 740 (monosubstituted benzene); MS: m/z 551 $\left(\mathrm{M}^{+}\right)$. Calculated for $\mathrm{C}_{28} \mathrm{H}_{24} \mathrm{Cl}_{2} \mathrm{CuN}_{4}: 60.98 \% \mathrm{C}$; $4.35 \% \mathrm{H} ; 10.16 \%$ N. Found: $60.96 \%$ C; $4.37 \% \mathrm{H}$; $10.14 \% \mathrm{~N}$; 3b: IR $\left(\mathrm{KBr}, \mathrm{cm}^{-1}\right): 3210(\mathrm{~N}-\mathrm{H}), 3015$ (Ar-H ), 2926 (C-H aliphatic), $1627(\mathrm{C}=\mathrm{N}), 1605$ $(\mathrm{C}=\mathrm{C}$ aromatic), $1255(\mathrm{C}-\mathrm{N}), 744(\mathrm{C}-\mathrm{Cl}) ; \mathrm{MS}: \mathrm{m} / \mathrm{z}$ $468\left(\mathrm{M}^{+}\right)$. Calculated for $\mathrm{C}_{16} \mathrm{H}_{14} \mathrm{Cl}_{4} \mathrm{CuN}_{4}: 41.02 \% \mathrm{C}$; $2.99 \% \mathrm{H} ; 11.96 \% \mathrm{~N}$. Found: $41.02 \% \mathrm{C} ; 2.97 \% \mathrm{H}$; $11.94 \% \mathrm{~N}$; 3c: IR (KBr, cm $\left.{ }^{-1}\right): 3258,3190(\mathrm{~N}-\mathrm{H})$, $3012($ Ar-H $), 1622(\mathrm{C}=\mathrm{N}), 1594(\mathrm{C}=\mathrm{C}$ aromatic), 1279 (C-N); MS: m/z $553\left(\mathrm{M}^{+}\right)$. Calculated for $\mathrm{C}_{26} \mathrm{H}_{22} \mathrm{Cl}_{2} \mathrm{CuN}_{6}: 56.42 \% \mathrm{C} ; 3.97 \% \mathrm{H} ; 15.18 \% \mathrm{~N}$. Found: $56.40 \% \mathrm{C}$; $3.95 \% \mathrm{H} ; 15.15 \% \mathrm{~N}$; 3d: IR $\left(\mathrm{KBr}, \mathrm{cm}^{-1}\right): 3218(\mathrm{~N}-\mathrm{H}), 3082(\mathrm{Ar}-\mathrm{H}), 1615(\mathrm{C}=\mathrm{N})$, $1595 \quad(\mathrm{C}=\mathrm{C}$ aromatic), $1274 \quad(\mathrm{C}-\mathrm{N}), \quad 748$ (monosubstituted benzene); MS: m/z $523\left(\mathrm{M}^{+}\right)$. Calculated for $\mathrm{C}_{26} \mathrm{H}_{20} \mathrm{Cl}_{2} \mathrm{CuN}_{4}: 39.65 \% \mathrm{C} ; 3.82 \%$ $\mathrm{H} ; 10.70 \% \mathrm{~N}$. Found: $39.64 \% \mathrm{C} ; 3.85 \% \mathrm{H} ; 10.68 \%$ $\mathrm{N}$; 3e: IR (KBr, $\left.\mathrm{cm}^{-1}\right)$ : $3348(\mathrm{~N}-\mathrm{H}), 3030(\mathrm{Ar}-\mathrm{H})$, $2565(\mathrm{SH}), 1622(\mathrm{C}=\mathrm{N}), 1605(\mathrm{C}=\mathrm{C}$ aromatic $), 1240$ (C-N); MS: $\mathrm{m} / \mathrm{z} 435 \quad\left(\mathrm{M}^{+}\right)$. Calculated for $\mathrm{C}_{14} \mathrm{H}_{12} \mathrm{Cl}_{2} \mathrm{CuN}_{4} \mathrm{~S}_{2}: 38.62 \% \mathrm{C} ; 2.75 \% \mathrm{H} ; 12.87 \% \mathrm{~N}$. Found: $38.60 \% \mathrm{C} ; 2.73 \% \mathrm{H} ; 12.89 \% \mathrm{~N}$; 3f: IR $\left(\mathrm{KBr}, \mathrm{cm}^{-1}\right): 3285(\mathrm{~N}-\mathrm{H}), 3020(\mathrm{Ar}-\mathrm{H}), 2928(\mathrm{C}-\mathrm{H}$ aliphatic), $1635(\mathrm{C}=\mathrm{N}), 1606(\mathrm{C}=\mathrm{C}$ aromatic $), 1286$ (C-N), 740 (monosubstituted benzene); MS: m/z 615 $\left(\mathrm{M}^{+}\right)$. Calculated for $\mathrm{C}_{28} \mathrm{H}_{24} \mathrm{Cl}_{2} \mathrm{CuN}_{4} \mathrm{~S}_{2}: 54.63 \% \mathrm{C}$; $3.90 \% \mathrm{H} ; 9.10 \%$ N. Found: $54.61 \%$ C; $3.93 \% \mathrm{H}$; $9.12 \% \mathrm{~N}$. 
The same procedure was applied for the preparation of other complexes $4 \boldsymbol{a}-\mathbf{f}$ 4a: IR $(\mathrm{KBr}, \mathrm{cm}$ $\left.{ }^{-1}\right)$ : $3225(\mathrm{~N}-\mathrm{H}), 3040$ (Ar-H ), 2865 (C-H aliphatic), $1621(\mathrm{C}=\mathrm{N}), 1603(\mathrm{C}=\mathrm{C}$ aromatic $), 1274(\mathrm{C}-\mathrm{N}), 746$ (monosubstituted benzene); MS: $\mathrm{m} / \mathrm{z} 546\left(\mathrm{M}^{+}\right)$. Calculated for $\mathrm{C}_{28} \mathrm{H}_{24} \mathrm{Cl}_{2} \mathrm{CoN}_{4}: 61.53 \% \mathrm{C} ; 4.39 \%$ H; $10.25 \%$ N. Found: $61.50 \%$ C; $4.41 \% \mathrm{H} ; 10.26 \%$ $\mathrm{N} ; 4 \mathbf{b}$ : IR (KBr, cm $\left.{ }^{-1}\right): 3260(\mathrm{~N}-\mathrm{H}), 3060(\mathrm{Ar}-\mathrm{H})$, 2980 (C-H aliphatic), $1624(\mathrm{C}=\mathrm{N}), 1604 \quad(\mathrm{C}=\mathrm{C}$ aromatic), $1289 \quad(\mathrm{C}-\mathrm{N}) . \quad \mathrm{MS}: \quad \mathrm{m} / \mathrm{z} \quad 464 \quad\left(\mathrm{M}^{+}\right)$. Calculated for $\mathrm{C}_{16} \mathrm{H}_{14} \mathrm{Cl}_{4} \mathrm{CoN}_{4}: 41.46 \% \mathrm{C} ; 3.02 \%$ H; $12.09 \%$ N. Found: $41.49 \%$ C; $3.03 \%$ H; $12.04 \%$ N; 4c: IR (KBr, cm $\left.{ }^{-1}\right): 3255,3198(\mathrm{~N}-\mathrm{H}), 3014$ (Ar$\mathrm{H}), 1624(\mathrm{C}=\mathrm{N}), 1610(\mathrm{C}=\mathrm{C}$ aromatic $), 1281(\mathrm{C}-\mathrm{N})$; MS: $\mathrm{m} / \mathrm{z} 548\left(\mathrm{M}^{+}\right)$. Calculated for $\mathrm{C}_{26} \mathrm{H}_{22} \mathrm{Cl}_{2} \mathrm{CoN}_{6}$ : $56.93 \% \mathrm{C} ; 4.01 \% \mathrm{H} ; 15.32 \% \mathrm{~N}$. Found: $56.95 \%$ C; $4.05 \% \mathrm{H} ; 15.30 \% \mathrm{~N}$; 4d: IR $\left(\mathrm{KBr}, \mathrm{cm}^{-1}\right): 3275$ (N-H), 3068 (Ar-H ), $1618(\mathrm{C}=\mathrm{N}), 1607(\mathrm{C}=\mathrm{C}$ aromatic), 1272 (C-N), 749 (monosubstituted benzene); MS: $\mathrm{m} / \mathrm{z} 518\left(\mathrm{M}^{+}\right)$. Calculated for $\mathrm{C}_{26} \mathrm{H}_{20} \mathrm{Cl}_{2} \mathrm{CoN}_{4}: 60.23 \% \mathrm{C} ; 3.86 \% \mathrm{H} ; 10.81 \% \mathrm{~N}$. Found: $60.26 \% \mathrm{C} ; 3.87 \% \mathrm{H} ; 10.79 \% \mathrm{~N}$; 4e: IR $\left(\mathrm{KBr}, \mathrm{cm}^{-1}\right): 3324(\mathrm{~N}-\mathrm{H}), 3059$ (Ar-H ), 2560 (SH), $1626(\mathrm{C}=\mathrm{N}), 1590(\mathrm{C}=\mathrm{C}$ aromatic $), 1265(\mathrm{C}-\mathrm{N})$; MS: $\mathrm{m} / \mathrm{z} 430\left(\mathrm{M}^{+}\right)$. Calculated for $\mathrm{C}_{14} \mathrm{H}_{12} \mathrm{Cl}_{2} \mathrm{CoN}_{4} \mathrm{~S}_{2}$ : $39.06 \% \mathrm{C} ; 2.79 \% \mathrm{H} ; 13.02 \% \mathrm{~N}$. Found: $39.09 \%$ C; $13.00 \% \mathrm{H} ; 3.03 \% \mathrm{~N}$; 4f: IR (KBr, $\left.\mathrm{cm}^{-1}\right): 3197$ (N-H), 3057 (Ar-H ), 2964 (C-H aliphatic), 1635 $(\mathrm{C}=\mathrm{N}), 1617,1601(\mathrm{C}=\mathrm{C}$ aromatic), $1269(\mathrm{C}-\mathrm{N})$; $\mathrm{MS}$ : $\mathrm{m} / \mathrm{z} 610\left(\mathrm{M}^{+}\right)$. Calculated for $\mathrm{C}_{28} \mathrm{H}_{24} \mathrm{Cl}_{2} \mathrm{CoN}_{4} \mathrm{~S}_{2}$ : $55.08 \%$ C; $3.93 \%$ H; $9.18 \%$ N. Found: $55.06 \%$ C; $3.96 \% \mathrm{H} ; 9.17 \% \mathrm{~N}$.

Synthesis of Dichloro[2-(alkyl/arylamine-1ylmethyl)-1H-benzimidazoles] copper(II) $\mathbf{3 g}$-i: Appropriate benzimidazole ligand 2g-i $(0.005 \mathrm{~mol})$ was dissolved in ethanol $(50 \mathrm{ml})$ and the resulting solution was treated with copper(II) chloride $(0.005$ mol) dissolved in dimethylformamide $(2 \mathrm{ml})$. The reaction mixture was stirred for $10 \mathrm{~h}$ at room temperature. The solid that separated in each was filtered, washed with ether, dried and recrystallized from dimethylformamide; $3 g$ : IR $\left(\mathrm{KBr}, \mathrm{cm}^{-1}\right): 3221$ (N-H), 3025 (Ar-H ), 2968, 2854 (C-H aliphatic), $1622(\mathrm{C}=\mathrm{N}), 1598(\mathrm{C}=\mathrm{C}$ aromatic $), 1275(\mathrm{C}-\mathrm{N})$; MS: $\mathrm{m} / \mathrm{z} 350\left(\mathrm{M}^{+}\right)$. Calculated for $\mathrm{C}_{13} \mathrm{H}_{17} \mathrm{Cl}_{2} \mathrm{CuN}_{3}: 44.57$ $\%$ C; $4.86 \% \mathrm{H} ; 12.00 \% \mathrm{~N}$. Found: $44.55 \% \mathrm{C} ; 4.88$ $\% \mathrm{H} ; 12.05 \% \mathrm{~N}$; 3h: IR (KBr, cm $\left.{ }^{-1}\right): 3280(\mathrm{~N}-\mathrm{H})$, 3030 (Ar-H ), 2980, 2860 (C-H aliphatic), 1642 $(\mathrm{C}=\mathrm{N}), 1615(\mathrm{C}=\mathrm{C}$ aromatic $), 1265(\mathrm{C}-\mathrm{N}) ; \mathrm{MS}: \mathrm{m} / \mathrm{z}$ $364\left(\mathrm{M}^{+}\right)$. Calculated for $\mathrm{C}_{14} \mathrm{H}_{19} \mathrm{Cl}_{2} \mathrm{CuN}_{3}: 46.15 \% \mathrm{C}$; $5.21 \% \mathrm{H} ; 11.54 \% \mathrm{~N}$. Found: $46.19 \% \mathrm{C} ; 5.19 \% \mathrm{H}$; $11.51 \% \mathrm{~N}$; 3i: IR $\left(\mathrm{KBr}, \mathrm{cm}^{-1}\right): 3202(\mathrm{~N}-\mathrm{H}), 3056$ (Ar-H ), 2921, 2850 (C-H aliphatic), $1629(\mathrm{C}=\mathrm{N})$, $1604(\mathrm{C}=\mathrm{C}$ aromatic), 1271 (C-N), 1112 (C-O-C); MS: $\mathrm{m} / \mathrm{z} 352\left(\mathrm{M}^{+}\right)$. Calculated for $\mathrm{C}_{12} \mathrm{H}_{15} \mathrm{Cl}_{2} \mathrm{CuN}_{3} \mathrm{O}$ : $40.90 \% \mathrm{C} ; 4.26 \% \mathrm{H} ; 11.93 \% \mathrm{~N}$. Found: $40.95 \%$ C; $4.24 \% \mathrm{H} ; 11.90 \% \mathrm{~N}$.

The same procedure was applied for the preparation of other complexes 4g-i. 4g: IR ( $\mathrm{KBr}$, $\mathrm{cm}^{-1}$ ): $3140(\mathrm{~N}-\mathrm{H}), 3015$ (Ar-H ), 2916 (C-H aliphatic), $1622(\mathrm{C}=\mathrm{N}), 1596(\mathrm{C}=\mathrm{C}$ aromatic $), 1250$ (C-N); MS: $\mathrm{m} / \mathrm{z} 345 \quad\left(\mathrm{M}^{+}\right)$. Calculated for $\mathrm{C}_{13} \mathrm{H}_{17} \mathrm{Cl}_{2} \mathrm{CoN}_{3}: 45.21 \% \mathrm{C} ; 4.92 \% \mathrm{H} ; 12.17 \% \mathrm{~N}$. Found: $45.20 \% \mathrm{C} ; 4.94 \% \mathrm{H} ; 12.15 \% \mathrm{~N}$; 4h: IR $\left(\mathrm{KBr}, \mathrm{cm}^{-1}\right): 3244(\mathrm{~N}-\mathrm{H}), 3032(\mathrm{Ar}-\mathrm{H}), 2958,2865$ (C-H aliphatic), $1643(\mathrm{C}=\mathrm{N}), 1608(\mathrm{C}=\mathrm{C}$ aromatic), 1268 (C-N); MS: m/z $359\left(\mathrm{M}^{+}\right)$. Calculated for $\mathrm{C}_{14} \mathrm{H}_{19} \mathrm{Cl}_{2} \mathrm{CoN}_{3}: 46.79 \% \mathrm{C} ; 5.29 \% \mathrm{H} ; 11.69 \% \mathrm{~N}$. Found: $46.76 \% \mathrm{C} ; 5.30 \% \mathrm{H} ; 11.67 \% \mathrm{~N}$; 4i: IR $\left(\mathrm{KBr}, \mathrm{cm}^{-1}\right): 3244(\mathrm{~N}-\mathrm{H}), 3028(\mathrm{Ar}-\mathrm{H}), 2924(\mathrm{C}-\mathrm{H}$ aliphatic), $1622(\mathrm{C}=\mathrm{N}), 1601(\mathrm{C}=\mathrm{C}$ aromatic $), 1232$ (C-N), 1118 (C-O-C). MS: m/z $347\left(\mathrm{M}^{+}\right)$. Calculated for $\mathrm{C}_{12} \mathrm{H}_{15} \mathrm{Cl}_{2} \mathrm{CoN}_{3} \mathrm{O}: 41.49 \% \mathrm{C} ; 4.32 \% \mathrm{H} ; 12.10 \%$ N. Found: $41.46 \% \mathrm{C} ; 4.30 \% \mathrm{H} ; 12.08 \mathrm{~N}$.

UV-Vis absorption study. UV-Vis absorption study of complexes $\mathbf{3 a}$ and $\mathbf{4 a}$ at $4 \mu \mathrm{M}$ concentration was carried out with respect to their corresponding ligands 1a and 4a, respectively in phosphate buffer saline solution (Dulbecco's buffer, pH 7.4), prewarmed at $37^{\circ} \mathrm{C}$ (Figure 1 and 2). $10 \mu 1$ of $20 \mathrm{mM}$ DMF solution of the copper (3a) and cobalt (4a) complexes was added separately to Dulbecco's buffer (pH 7.4), pre-warmed at $37^{\circ} \mathrm{C}$, resulting in a final concentration of $4 \mu \mathrm{M}$. Spectra at $0,1,2$ and $3 \mathrm{~h}$ were recorded between 240 and $400 \mathrm{~nm}$. The resulting stability profile is shown in figure 3 and 4 . 


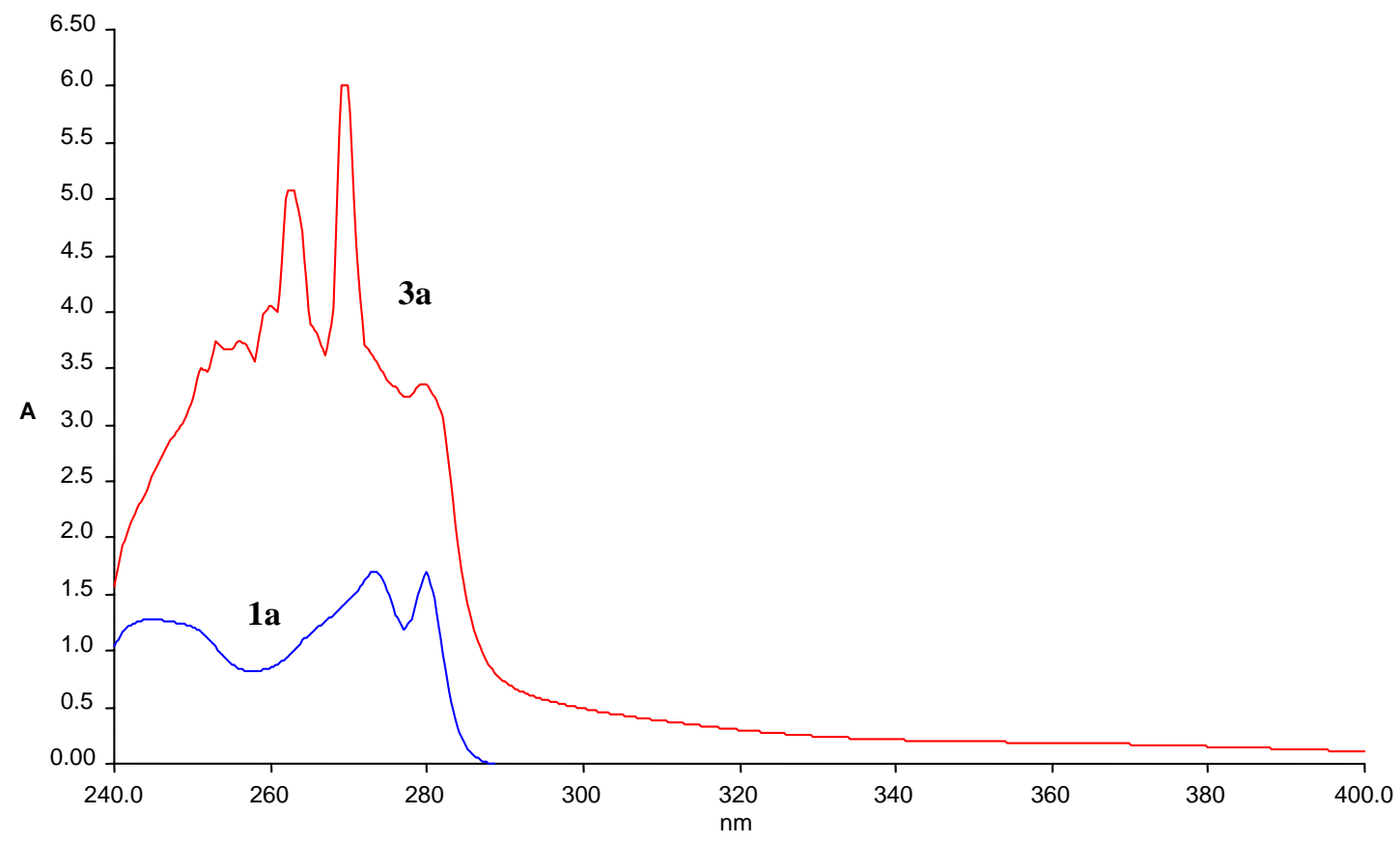

Figure 1. UV profile of compounds 1a and 3a in Dulbecco's buffer $\left(\mathrm{pH} 7.4,37^{\circ} \mathrm{C}\right)$

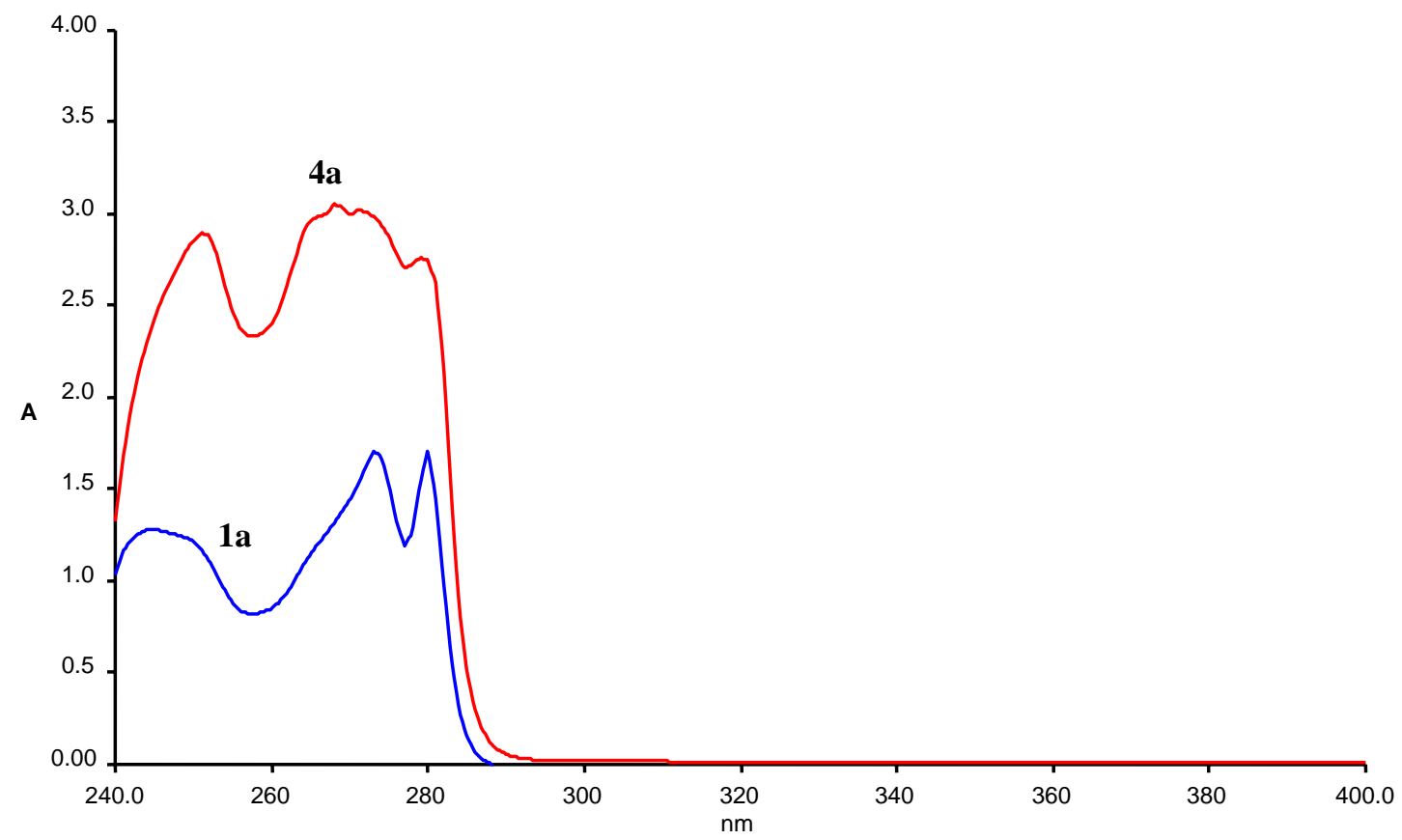

Figure 2. UV profile of compounds 1a and 4a in Dulbecco's buffer (pH 7.4, $\left.37{ }^{\circ} \mathrm{C}\right)$ 


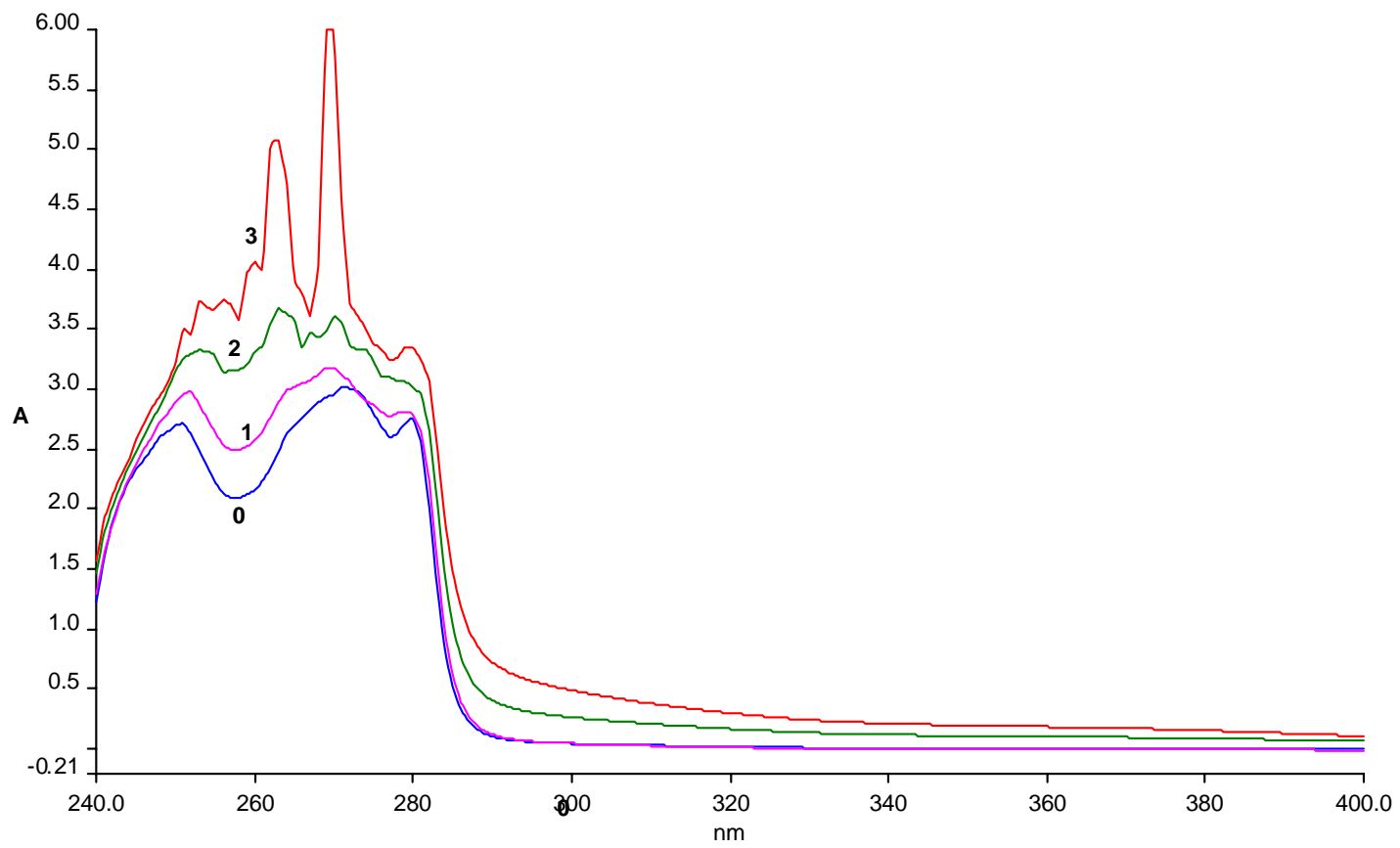

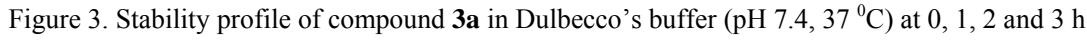

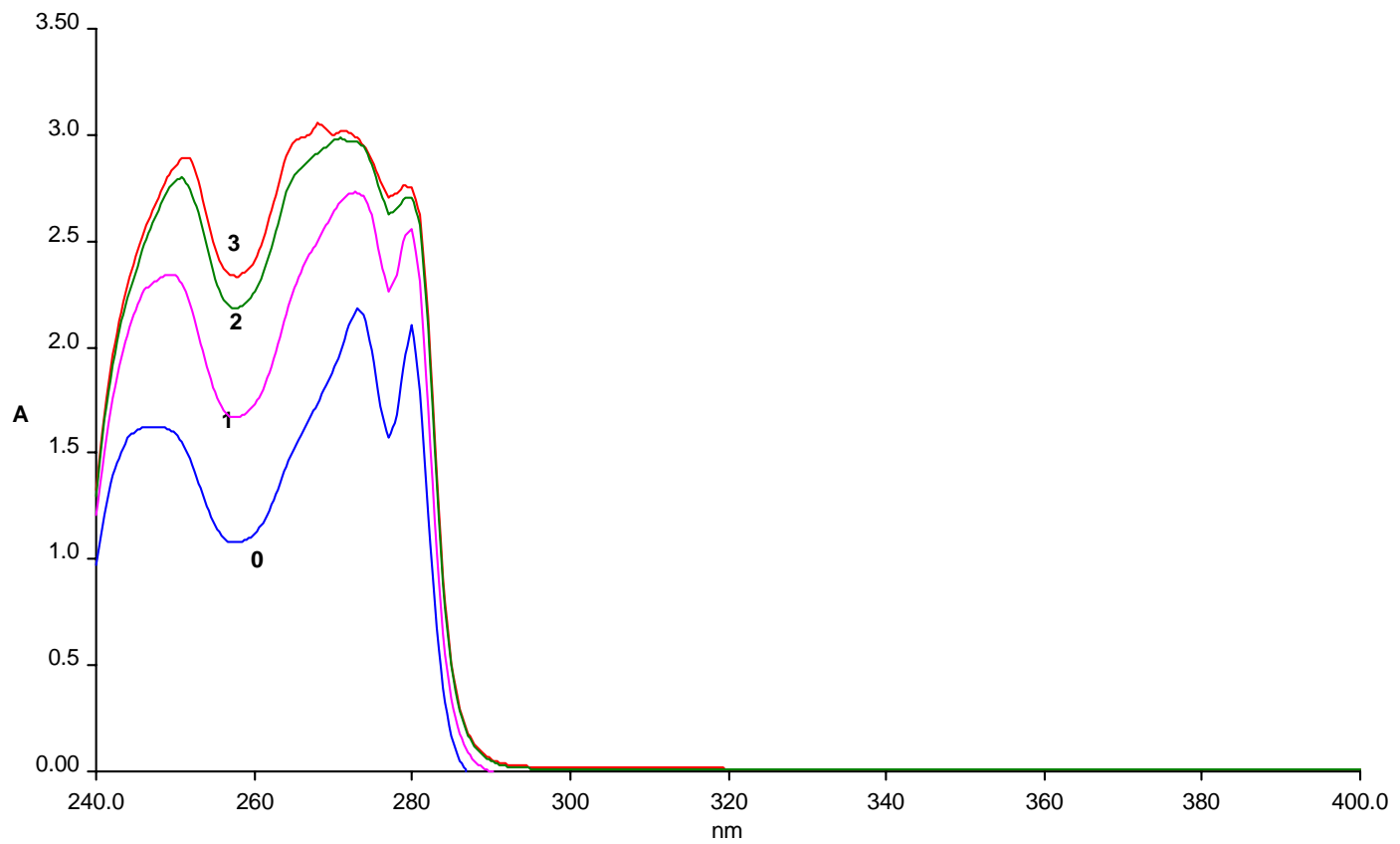

Figure 4. Stability profile of compound 4a Dulbecco's buffer $\left(\mathrm{pH} 7.4,37^{\circ} \mathrm{C}\right)$ at $0,1,2$ and $3 \mathrm{~h}$ 
Biological evaluation. DPPH radical scavenging assay: Compounds 3a-i and 4a-i were evaluated as DPPH (1,1-diphenyl-2-picryl hydrazyl) free radical scavengers as described by Hwang et. al. ${ }^{18} 10 \mu \mathrm{l}$ of the compound solution in a $1: 1 \mathrm{DMSO} /$ phosphate buffer (PH 7.4) mixture and $0.2 \mathrm{ml}$ of $\mathrm{DPPH}$ in methanol solution was incubated at $37{ }^{\circ} \mathrm{C}$ for $30 \mathrm{~min}$ and the absorbance of the supernatant was measured at $490 \mathrm{~nm}$. $\mathrm{IC}_{50}$ values were determined for the complexes $3 \mathrm{a}-\mathrm{i}$ and $4 \mathrm{a}-\mathrm{i}$ and the result is summarized with reference standard ascorbic acid in Table 2.

Nitric oxide radical inhibition assay: Nitric oxide radical inhibition assay of compounds 3a-i and 4a-i was assayed $^{19}$ by incubating $2 \mathrm{ml}$ sodium nitroprusside $(10 \mathrm{mM}), 0.5$ phosphate buffer saline and $0.5 \mathrm{ml}(0.25 \mathrm{mg})$ of compound solution in a $1: 1$ DMSO/phosphate buffer (PH 7.4) mixture at $25{ }^{\circ} \mathrm{C}$ for $150 \mathrm{~min}$. Then $1 \mathrm{ml}$ of sulfanilic acid reagent was added to $0.5 \mathrm{ml}$ of reaction mixture for $5 \mathrm{~min}$ to complete diazotization. Subsequently, $1 \mathrm{ml}$ naphthyl ethylene diamine dihydrochloride (NEDD) was added and allowed to stand for $30 \mathrm{~min}$ at $25{ }^{\circ} \mathrm{C}$. The absorbance of these solutions was measured at 540 $\mathrm{nm}$. $\mathrm{IC}_{50}$ values were determined for the complexes 3a-i and 4a-i and the result is summarized with reference standard ascorbic acid in Table 2.

Superoxide dismutase (SOD) activity: Super oxide dismutase (SOD) activity of complexes 3a-i and 4a-i was determined in a 96 well microtitre plate assay based on the ability of the complexes to inhibit the reduction of nitroblue tetrazolium salt (NBT) by superoxide ions, which are generated by the traditional xanthine/xanthine oxidase system $^{20}$. Superoxide radical anions reduce NBT to a blue formazan, which was detected spectrophotometrically at $570 \mathrm{~nm}$. Solutions of the synthesized compounds at four different concentrations were prepared in a 1:1 DMSO/phosphate buffer (PH 7.4) mixture and $10 \mu \mathrm{l}$ were added to 8 wells per concentration to give a concentration range between 0.01 and $1 \mu \mathrm{M}$. Atleast three independent assays were performed for each complex and $\mathrm{IC}_{50}$ values were calculated for the complexes 3a-i and $\mathbf{4 a - i}$ and the result is summarized with reference standard rutin in Table 2.

Table 2. Anticancer and antioxidant activity of compounds $4 a-i$ and $5 a-i$

\begin{tabular}{|c|c|c|c|c|c|}
\hline \multirow[t]{2}{*}{ Compound } & \multicolumn{3}{|c|}{ *Antioxidant activity } & \multicolumn{2}{|c|}{$\begin{array}{l}\text { ** The average percent cell growth } \\
\text { values }\end{array}$} \\
\hline & $\begin{array}{c}\text { DPPH } \\
\mathrm{IC}_{50}(\mu \mathrm{g} / \mathrm{ml})\end{array}$ & $\begin{array}{l}\text { Nitric oxide } \mathrm{IC}_{50} \\
\quad(\mu \mathrm{g} / \mathrm{ml})\end{array}$ & $\begin{array}{l}\text { SOD activity } \\
\mathrm{IC}_{50}(\mu \mathrm{M})\end{array}$ & MCF 7 cell line & $\begin{array}{l}\text { SF } 268 \text { cell } \\
\text { line }\end{array}$ \\
\hline $3 a$ & 51 & $>500$ & 0.65 & 0.00 & 15.43 \\
\hline $3 b$ & $>500$ & 211 & 0.98 & 79.98 & 85.35 \\
\hline $3 \mathrm{c}$ & $>500$ & 250 & 0.62 & 34.44 & 68.44 \\
\hline $3 d$ & $>500$ & 200 & 0.90 & 94.44 & 83.67 \\
\hline $3 e$ & $>500$ & $>500$ & 0.59 & 77.50 & 78.23 \\
\hline $3 f$ & $>500$ & $>500$ & 0.47 & 43.55 & 78.55 \\
\hline $3 g$ & $>500$ & 196 & 0.34 & 97.30 & 96.45 \\
\hline $3 \mathrm{~h}$ & $>500$ & $>500$ & 0.44 & 97.40 & 98.55 \\
\hline $3 \mathrm{i}$ & $>500$ & 76 & 0.26 & 97.14 & 80.25 \\
\hline $4 a$ & $>500$ & 65 & 0.32 & 56.54 & 75.65 \\
\hline $4 \mathrm{~b}$ & $>500$ & 165 & 0.56 & 44.54 & 56.25 \\
\hline $4 \mathrm{c}$ & $>500$ & $>500$ & 0.43 & 12.43 & 60.68 \\
\hline $4 d$ & $>500$ & $>500$ & 0.52 & 24.65 & 55.68 \\
\hline $4 e$ & $>500$ & $>500$ & 0.49 & 98.55 & 92.35 \\
\hline $4 \mathrm{f}$ & $>500$ & $>500$ & 0.48 & 55.23 & 56.25 \\
\hline $4 \mathrm{~g}$ & $>500$ & $>500$ & 0.28 & 12.33 & 15.35 \\
\hline $4 \mathrm{~h}$ & $>500$ & $>500$ & 0.58 & 58.23 & 60.55 \\
\hline $4 \mathrm{i}$ & $>500$ & $>500$ & 0.65 & 66.21 & 70.35 \\
\hline $\begin{array}{l}\text { Standard } \\
\text { drug }\end{array}$ & $\begin{array}{c}14 \\
\text { (Ascorbic acid) }\end{array}$ & $\begin{array}{c}69 \\
\text { (Rutin) }\end{array}$ & $\begin{array}{c}0.45 \\
\text { (Rutin) }\end{array}$ & --- & --- \\
\hline
\end{tabular}

* Average of three determinations. **Average of three determinations at $20 \mu \mathrm{M}$ against human breast MCF 7 and CNS SF 268 cancer cell lines, zero means all cells are dead, the untreated controls showed $100 \%$ growth. 
In vitro antitumor activity: Antitumor activity of the newly synthesized compounds were evaluated by tryphan blue dye exclusion technique ${ }^{21}$ against human breast MCF-7 and CNS SF 268 cancer cell lines at the concentration level of $20 \mu \mathrm{M}$. Each cell line is preincubated on microtitre plate, the test compounds are then added at $20 \mu \mathrm{M}$ concentration. Cultures are incubated for fourty eight hours. Results for each compound are reported as the percent growth of the treated cells when compared to the untreated control cells. Primary screening of the compounds was done to indicate whether a compound possessed enough activity at this concentration to inhibit cell growth by $50 \%$. Results are given in Table 2.

\section{RESULTS AND DISCUSSION}

The required starting material 2-substituted-1Hbenzimidazoles 1a-f was prepared ${ }^{17}$ by refluxing a mixture of $o$-phenylenediamine dihydrochloride and suitable aliphatic/aromatic carboxylic acid. Compounds 1a-f when stirred with copper(II) and cobalt(II) chlorides separately in aqueous ethanol resulted in the formation of dichlorobis[2-substituted benzimidalzoles] copper(II) 3a-f and dichlorobis [2substituted benzimidazoles] cobalt(II) 4a-f, respectively. 2-Chloromethyl-1H-benzimidazole $\mathbf{1 b}$ was condensed with appropriate amine in dry acetone to furnish 2-(amino-1-ylmethyl)-1H-benzimidazoles 2g-i. Further treatment of 2g-i with copper(II) chloride and cobalt(II) chloride yielded dichloro[2(amino-4ylmethyl)-1H-benzimidazoles] copper(II) 3g-i and dichloro[(2-(amino-4ylmethyl)-1Hbenzimidazoles] cobalt(II) $4 \mathbf{g}$-i, respectively. All the ligands synthesized were characterized by their IR, mass, ${ }^{1} \mathrm{H}$ NMR spectroscopy and elemental analysis. Metal complexes synthesized were characterized by IR, UV and elemental analysis. Elemental analysis suggested 1:2 (metal:ligand) stoichiometry for $\mathbf{3 a - i}$ and 4a-i and 1:1 (metal : ligand) stoichiometry for 3g-i and 4g-i complexes. IR spectrum of the complexes has shown some characteristic changes when compared to the free ligands. Ligands showed broad bands in the region $3460-3340 \mathrm{~cm}^{-1}$ due to the intermolecular hydrogen bonded imidazole $\mathrm{N}-\mathrm{H}$ stretching. All the complexes which have free imidazole N-H, exhibited N-H stretching bands ranging from 3322 to $3140 \mathrm{~cm}^{-1}$ sharper than those of the ligands due to breaking of tautomerism, indicating that imidazole N-H was not involved in the coordination. Benzimidazole ligands exhibit strong to medium intensity bands in the region $1665-1630 \mathrm{~cm}^{-}$ ${ }^{1}(\mathrm{C}=\mathrm{N}$ stretching), undergo a negative shift of 10-25 $\mathrm{cm}^{-1}$ which indicates involvement of azomethine nitrogen upon complexation. The other bands in the spectrum of each complex were similar to those in the corresponding ligand spectrum except for slight shifts in their positions and changes in their intensities due to coordination.

Figure 1 and 2 presents representative UV-Vis spectra of two pairs of ligands and complexes. The absorptions in the UV region are comparable for both the ligands and complexes of copper(II) and cobalt(II). The absorptions of the copper(II) and cobalt(II) complexes, however, extend further out into the visible region $>380 \mathrm{~nm}$. Fig. 3 and 4 show the time-dependant changes in the UV-Vis spectra of the two of the complexes over $3 \mathrm{~h}$ when they were incubated at $37{ }^{\circ} \mathrm{C}$, in phosphate buffered saline. Compounds 3a and 4a showed a slow decrease in the intensity of the shape of the spectra over $3 \mathrm{~h}$ incubation but no change in the shape of the spectra and no crossing of the spectra was observed.

It is evident from result (Table 2) that only Copper(II) complex 3a exhibited moderate $\left(\mathrm{IC}_{50} 51\right.$ $\mu \mathrm{g} / \mathrm{ml})$ DPPH free radical scavenging activity while all other compounds were found to be inactive $\left(\mathrm{IC}_{50}\right.$ $>500 \mu \mathrm{g} / \mathrm{ml}$ ) in comparison to the reference standard ascorbic acid $\left(\mathrm{IC}_{50} 41 \mu \mathrm{g} / \mathrm{ml}\right)$. In nitric oxide method compounds $\mathbf{3 i}$ and $4 \mathbf{a}$ showed significant free radical scavenging activity with an $\mathrm{IC}_{50}$ of $76 \mu \mathrm{g} / \mathrm{ml}$ and 65 $\mu \mathrm{g} / \mathrm{ml}$, respectively in comparison to the reference standard rutin $\left(\mathrm{IC}_{50} 69 \mu \mathrm{g} / \mathrm{ml}\right)$. Compounds $\mathbf{3 b}, \mathbf{3 c}$, 3d, $\mathbf{3 g}$ and $\mathbf{4 b}$ exhibited moderate activity while all other compounds were found to be inactive and no correlation was observed between nitric oxide free 
radical scavenging activity, different substituents and $\mathrm{Cu}$ (II) and $\mathrm{Co}(\mathrm{II})$ complexes.

It is evident from superoxide dismutase activity result (Table 2) that compounds $\mathbf{3 f}-\mathbf{i}, \mathbf{4 a}, \mathbf{4 c}$ and $\mathbf{4 e - g}$ exhibited potent activity $\left(\mathrm{IC}_{50} 0.26-0.49 \mu \mathrm{M}\right)$ while all other compounds were found to be moderately active in comparison to the reference standard rutin $\left(\mathrm{IC}_{50} 0.45 \mu \mathrm{M}\right)$. Copper(II) complex $3 \mathbf{i}$ bearing morpholinomethyl group ( $\mathrm{IC}_{50} 0.26 \mu \mathrm{M}$ ) and co(II) complex $\mathbf{4 g}$ bearing piperidinomethyl group $\left(\mathrm{IC}_{50}\right.$ $0.28 \mu \mathrm{M}$ ) showed maximum superoxide dismutase activity.

As shown in Table 2 metal complexes 3a, 3c, 3f, 4b-d and $\mathbf{4 g}$ exhibited significant activity against human breast cancer MCF-7 cell line. Copper(II) complex bearing benzyl (3a) and cobalt(II) complexes bearing p-aminophenyl (4c) or piperidinomethyl $(\mathbf{4 g})$ substituents at position 2 of benzimidazole ring showed potent cytotoxicity against both human breast MCF 7 and human CNS SF 268 cancer cell lines while complexes 3c, $\mathbf{4 b}$ and 4d exhibited moderate cytotoxicity against human breast cancer cell lineMCF-7. As evident from result no correlation was observed between corresponding superoxide dismutase (SOD) $\mathrm{IC}_{50}$ values and cytotoxic activity. This indicates that mechanisms other than SOD mimicking activity are responsible for their cytotoxic properties. Copper(II) complex $\mathbf{3 i}$ showed significant SOD mimicking activity but no cytotoxicity on MCF-7 and SF 268 cancer cell lines at $20 \mu \mathrm{M}$ might be useful therapeutically as a SOD mimicking agent.

\section{ACKNOWLEDGEMENT}

The authors wish to place their regards to His Holiness Jagadguru Sri Sri Sri Shivarathri Deshikendra Mahaswamigalavaru of Sri Suttur mutt, Mysore for providing facilities.

\section{REFERENCES}

1. Devinder, M.R.J., Michael, B.R. and Sean, M.K. 2002. Synthesis and evaluation of anticancer benzoxazoles and benzimidazoles related to UK-1. Bioorg. Med. Chem. 10, 3997-4004.
2. Hong, S.Y., Chung, K.H., You, J.H., Choi, I.H., Chae, M.J., Han, J.Y., Jung, O.K., Kang, S.J. and Ryu, C.K. 2004. Synthesis and biological evaluation of benzimidazole-4,7diones that inhibit vascular smooth muscle cell proliferation. Bioorg. Med. Chem. Lett. 14, 3563-3566.

3. Patel, P.D., Patel, M.R., Kausik, B.N. and Talele, T.T. 2008. 3D QSAR and molecular docking studies of benzimidazole derivatives as hepatitis C virus NS5B polymerase inhibitors. J. Chem. Inf. Model. 48, 42-45.

4. Lopez, V.F., Medina-Franco, J.L., Hernandez-Campos, A., Rodriguez-Morales, S., Yepez, L., Cedillo, R. and Castillo, R. 2007. Molecular modeling of some 1H-benzimidazole derivatives with biological activity against Entamoeba histolytica: a comparative molecular field analysis study. Bioorg. Med. Chem. 15, 1117-1126.

5. Starcevic, K., Kralj, M., Eeter, K., Ssbel, I., Grce, M., Panelic, K. and Zamola, G.K. 2007. Synthesis, antiviral and antitumor activity of 2-substituted-5-amidinobenzimidazoles. Bioorg. Med. Chem. 15, 4419-4426.

6. White, A.W., Curtin, N.J., Eastman, B.W., Golding, B.T., Hostomsky, Z., Kyle, S., Li. J., Maegley, K.A., SkaL Itzky, D.J., Webber, S.E., Yu , X.H. and Griffin R. 2004. Potentiation of cytotoxic drug activity in human tumour cell lines, by amine substituted 2-arylbenzimidazole-4carboxamide PARP-1 inhibitors. Bioorg. Med. Chem. Lett. 14, 2433-2437.

7. Rangarajan, M, Kim, S., Jin, S., Sim, S. P., Liu, A., Pilch, D. S., Liu, L. F., La Voie, E. J. 2000. 2"-Substituted 5phenylterbenzimidazoles as topoisomerase I poisons. Bioorg. Med. Chem. 8, 1371-1382.

8. Farukh, M. B. and Shamim, A. 2005. Synthesis, antibacterial, antifungal activity and interaction of CT-DNA with a new benzimidazole derived $\mathrm{Cu}(\mathrm{II})$ complex. Eur. J. Med. Chem. 40, 1103-1110.

9. Roth, T., Eckert, C., Fiebig, H.H., Jung, M. 2002. Comparative action of cobalt carbonyl complexes on cancer cells using human tumor xenografts. Anticancer Res. 22, 2281-2284

10. Utku, S., Gumus, F., Gur, S., Ozkul, A. 2007. Synthesis and cytotoxic activity of platinum(II) and platinum(IV) complexes with 2-hydroxymethylbenzimidazole or 5(6)chloro-2-hydroxymethylbenzimidazole ligands against MCF7 and HeLa cell lines. Turk. J. Chem. 31, 503-514.

11. Gocke, M., Utku, S., Gur, S., Ozkul, A. and Gumus, F. 2005. Synthesis, in vitro cytotoxic and antiviral activity of cis$\left[\operatorname{Pt}(\mathrm{R}(-) \text { and } \mathrm{S}(+)-2-\alpha \text {-hydroxybenzylbenzimidazole })_{2} \mathrm{Cl}_{2}\right]$ complexes. Eur. J. Med. Chem. 40, 135-141. 
12. Saczewski, F., Dziemidowicz-Borys, E., Bednarski, P. J., Grünert, R., Gdaniec M., Tabin, P. 2006. Synthesis, crystal structure and biological activities of copper(II) complexes with chelating bidentate 2-substituted benzimidazole ligands. J. Inorg. Biochem. 100, 1389-1398.

13. Dionisi, D., Galeotti, T., Terranove, T., and Azzi, A. 1975. Superoxide radicals and hydrogen peroxide formation in mitochondria from normal and neoplastic tissues. Biochim. Biophys. Acta. 403, 292-300.

14. Sun Y. 1990. Free radicals, antioxidant enzymes and carcinogenesis. Free Radic. Biol. Med. 16, 275-282.

15. Farmer, K.J. and Sohal, R.S. 1989. Relationship between superoxide anion radical generation and aging in the housefly, Mucosa domestica. Free Radic. Biol. Med. 7, 2329.

16. Oberley, L.W. and Buettner, G.R. 1979. Role of superoxide dismutase in cancer. Cancer Res. 39, 1141-1149.

17. Furniss, B.S., Hannaford , A.J., Smith, P.W.G., Tatchell, A.R. 2005. In: Vogel's Text Book of Practical Organic Chemistry. $5^{\text {th }}$ Edn. Pearson Education Pvt. Ltd., Singapore, p.1162.
18. Hwang, B.Y., Kim, H.B., Lee, J.H., Hong, Y. S., RO, J. S., Lee, K.S. and Lee, J.J. 2001. Antioxidant benzoylated flavan3-ol glycoside from Celastrus orbiculatus. J. Nat. Prod. 64, 82-84.

19. Govindarajan, R., Rastogi, S., Vijaykumar, M., Rawat, A.K.S., Shriwaikar, A., Mehrotra, S., Puspangadam, P. Antioxidant potential of Anogeissus latifolia. 2004. Biol. Pharm. Bull. 27, 1266-1269.

20. McCord, J. M. and Fridovich, I. 1969. Superoxide dismutase. An enzymatic function for erythrocuprein (hemocuprein). $J$. Biol. Chem. 244, 6049-6055.

21. Bracht, K, Grunert, B.R. and Bednarski, P.J. 2006. Correlations between the activities of 19 anti-tumor agents and the intracellular glutathione concentrations in a panel of 14 human cancer cell lines: comparisons with the National Cancer Institute data. Anti-Cancer Drugs 17, 41-52.

22. Dictionary of Organic Compounds, $5^{\text {th }}$ and $6^{\text {th }}$ edn., Chapman and Hall: London, 1982 and 1997. 